\title{
Aspects of the Homogeneous Sine-Gordon models
}

\author{
Patrick Dorey \\ Department of Mathematical Sciences \\ University of Durham \\ Durham DH1 3LE, UK \\ E-mail: p. e.dorey@dur.ac.uuㅜㄴ \\ and J. Luis Miramontes* \\ Departamento de Física de Partículas \\ Universidad de Santiago de Compostela \\ 15782 Santiago de Compostela, Spain \\ E-mail: miriramont@usc.esi
}

Abstract: The physical mass scales that determine the behaviour of general (simplylaced) Homogeneous Sine-Gordon models are investigated by means of a study of their finite-size effects, using the thermodynamic Bethe ansatz. These models describe integrable multiparameter perturbations of the theory of level- $k G$-parafermions, where $G$ is a Lie group. The parameters can be related to adjustable mass scales of stable and unstable particle states. Our results confirm the presence of unstable particle states at generic values of $k$, as predicted at large $k$ by semiclassical arguments.

In this talk, we review some recent work on the multiparameter nature of a family of two-dimensional integrable quantum field theories called the Homogeneous sine-Gordon (HSG) models. More details will be presented in [1] [1]

\section{Introduction}

It is often productive to consider a quantum field theory as a perturbation of its ultraviolet limit. In two dimensions this approach is particularly effective, because the unperturbed theory is expected to be a conformal field theory, and two-dimensional conformal field theories are very well-understood. The power of this approach was first fully appreciated by A.B. Zamolodchikov. His $c$-theorem established non-perturbatively the monotonic nature of renormalisation group flows in unitary two-dimensional quantum field theories, and provided an important tool - the $c$-function - for the analysis of these flows [2]. As a first application, he was able to show that the perturbation of the minimal conformal

\footnotetext{
${ }^{*}$ Speaker.
} 
field theory $\mathcal{M}_{p, p+1}$ by its $\phi_{13}$ operator could induce a flow to the neighbouring theory $\mathcal{M}_{p-1, p}[\overline{3}]$. This result was obtained by combining the non-perturbative $c$-theorem with the fact that the separation of the two fixed points vanishes in the limit $p \rightarrow \infty$. The treatment, perturbative in $1 / p$, was in this respect analogous to Wilson's $\varepsilon$ expansion near to four dimensions.

To go further requires a second key observation of Zamolodchikov's, namely that some perturbations of conformal field theories break their infinite-dimensional conformal sym-

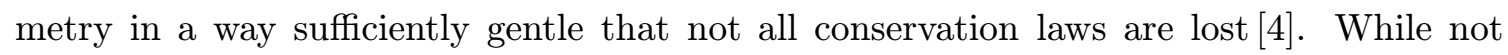
being scale invariant, the resulting models should still be integrable. With the aid of such theories one can hope to place disconnected fixed points of the renormalisation group within a web of exactly-solvable interpolating theories, thereby giving a much more detailed understanding of the set of all two-dimensional quantum field theories.

Given an integrable quantum field theory, it is natural to look for exact, nonperturbative techniques for the analysis of its renormalisation group flow. The exact S-matrix is insufficient for this task, related as it is to the long-distance (on-shell) properties of the theory. However, an S-matrix should characterise a model completely, and once it is known exactly, there are various ways to extract off-shell data. One of these is the formfactor expansion of correlation functions. In principle this allows the correlators entering Zamolodchikov's definition of his $c$-function (or those appearing in the integral representation derived by Cardy [in the RG flow can be analysed through the evolution of $c_{\mathrm{zam}}(r)$, as $r$ varies from 0 to $\infty$. However its very name gives away the fact that the form-factor technique provides an $e x$ pansion for correlation functions, and in practice it is very hard to go beyond the first four to six terms, even with the help of Monte Carlo methods to evaluate the necessary multidimensional integrals. Only very rarely (in cases which in some sense are 'free' in the relevant limit) is it possible to resum the series with sufficient precision to characterise exactly the deep ultraviolet behaviour.

For this reason, an alternative technique, called the 'thermodynamic Bethe ansatz', or TBA, has become popular. First introduced into the context of relativistic continuum field theory by Al.B. Zamolodchikov in [6] effective central charge $c_{\text {eff }}(r)$, which is defined as follows. Let $M$ be a mass scale in the perturbed theory - either the mass of an asymptotic state, or the inverse of a crossover scale if the theory is again massless in the far infrared. Now suppose that the one-dimensional space on which the model is defined is rolled up into a circle. The spectrum becomes discrete and all energy levels depend on the circumference $R$ of the circle. In particular, this is true for the ground state energy, which on general grounds should have the form

$$
E(R)=\mathcal{E} R-\frac{\pi}{6 R} c_{\text {eff }}(M R)
$$

where $\mathcal{E} R$ is a 'bulk' contribution proportional to the system size, and the more subtle parts of the $R$-dependence have been wrapped up in the dimensionless function $c_{\text {eff }}(M R)$, the 'effective central charge'. The name comes from the fact that, if a theory is conformal, then $c_{\text {eff }}$ is a constant, equal (in unitary theories) to the central charge of the theory [i] $]$. Thus $c_{\text {eff }}(r)$ has most of the useful properties of Zamolodchikov's $c$-function $c_{\mathrm{zam}}(r)$, though it 
is important to appreciate that away from the conformal points, the two functions are not the same. Since we'll be concentrating on the effective central charge in this article, from now on we will simply denote it as $c(r)$.

A definition such as (1.1) is all very well, but without a practical way to calculate $c(r)$, it is of little practical use. Fortunately Al.B. Zamolodchikov has shown how to apply general ideas of the thermodynamics of systems solvable by the Bethe ansatz to relativistic systems for which an exact S-matrix is known [i, equations, now called 'TBA equations'. Once these equations have been solved for a set of functions known as pseudoenergies, the effective central charge can be extracted as an integral. Specific examples will be seen later in the paper. While exact solutions of the TBA equations are hard to come by, their numerical treatment is very straightforward, and limiting behaviours can be obtained analytically. To give the reader a flavour of the results that can be obtained using the TBA method, in the remainder of this section we sketch some of the simple and not-so-simple behaviours that have been found over the last few years.

First of all, if a model is massive in the far infrared, then the $1 / R$ 'correction' term to the bulk behaviour of $(\overline{1}-1,1)$ must be absent in that limit, and so $c(r)$ must tend to zero as $r \rightarrow \infty$. Conversely, in the deep ultraviolet $c(r)$ will tend to the central charge $c_{U V}$ of the unperturbed conformal field theory, and in the most simplest situations $c(r)$ just interpolates between these two values as $\log (r)$ varies from $-\infty$ to $+\infty$, as illustrated in figure ${ }_{-1}^{1}$. This was the behaviour seen in [6] for the Lee-Yang and three-state Potts models; subsequently, the method has been applied to many other theories.
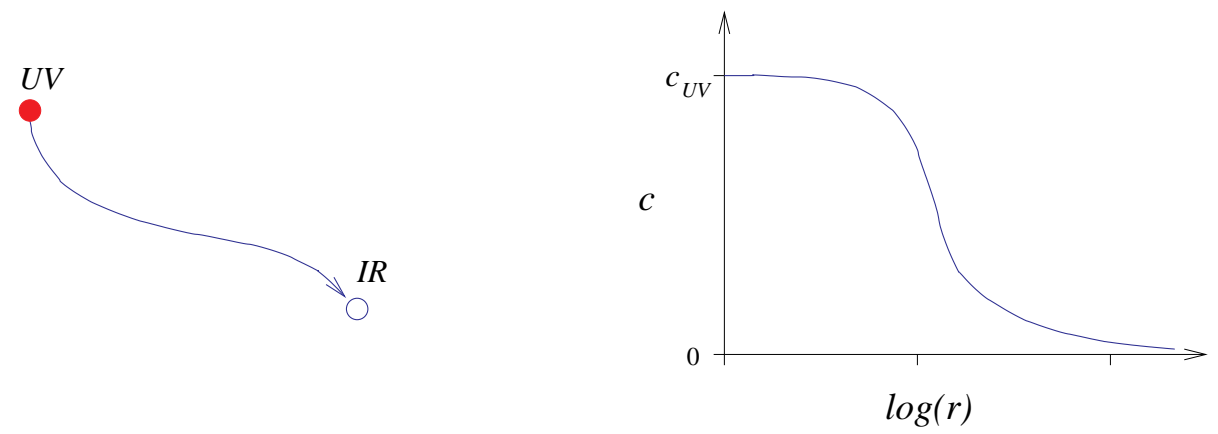

Figure 1: The RG flow and effective central charge for a typical massive perturbed conformal field theory. The filled dot denotes a fixed point of the RG with infinite correlation length; the empty dot, a 'massive' fixed point with zero correlation length.

For models with massless infrared limits, which interpolate between conformal field theories in the manner of the $\phi_{13}$ perturbation of $\mathcal{M}_{p, p+1}$ already mentioned above, the TBA machine is a little harder to use. The first examples of massless TBA systems were conjectured, for the interpolating $\phi_{13}$ flows, by Al.B. Zamolodchikov in [8]; ; they served to give nonperturbative confirmation that the picture of the flows developed by A.B. Zamolodchikov for large $p$ holds in general. Again, the TBA equations yield a function $c(r)$ interpolating between the expected values, in these cases the central charges $c_{U V}$ and $c_{I R}$ of the ultraviolet and infrared limiting models. The general picture is shown in figure $\overline{2}$, 

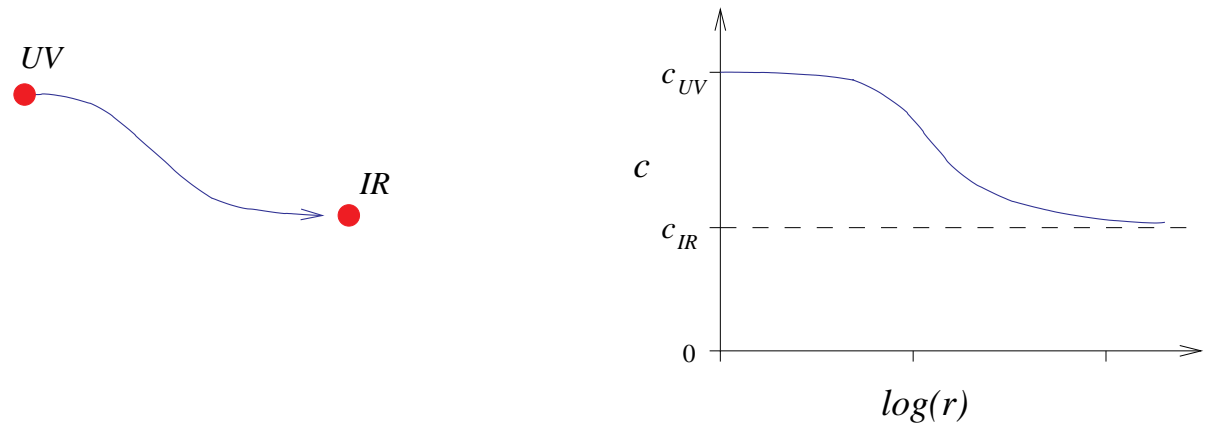

Figure 2: A typical massless flow. Labelling conventions as in figure

The next development was less expected. In [9.9] $]$, Al.B. Zamolodchikov pointed out a particularly simple TBA system, related by analytic continuation to that of the sinhGordon model, for which the flow pattern of the effective central charge was as illustrated on the right-hand side of figure $\underline{\underline{\beta}}$.
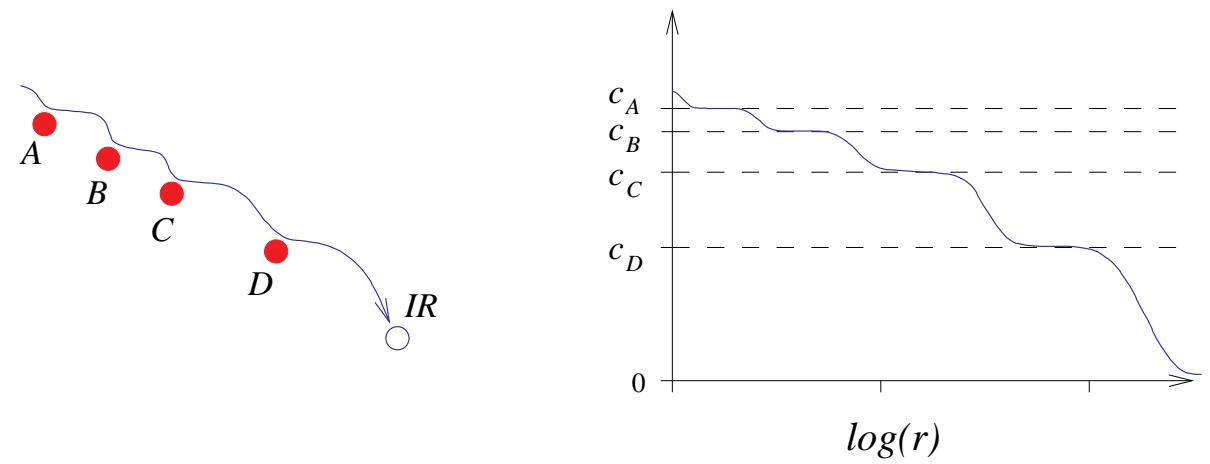

Figure 3: A (massive) 'staircase' flow, passing close to the RG fixed points $A, B, C$ and $D$. Labelling conventions as in figure

Over an infinite set of intermediate distance ranges, the effective central charge stabilises at an approximately constant 'plateau' value. For the system introduced in [i9i]', the plateau values are precisely the central charges of the minimal models $\mathcal{M}_{p, p+1}$; the natural interpretation is that the effective central charge produced by Zamolodchikov's TBA is that of an integrable quantum field theory whose RG trajectory passes close by the fixed points of all of the minimal models in turn, as shown on the left-hand half of figure ${ }_{-1}^{3}$. The conjectured theory is called a staircase model, for reasons which should be obvious on examining the figure. Subsequent work has generalised this in a number of

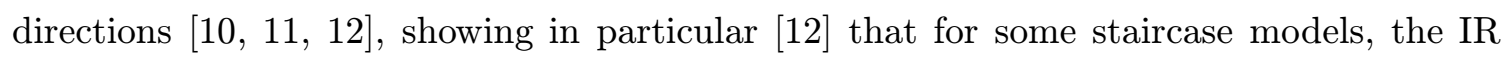
limit is massless. A couple of other properties are worth noting: first, the treatment of the deep UV limits of staircase models is rather subtle; and second, each staircase model in fact sits in a one-parameter family of such models, the parameter controlling how closely each fixed point is visited on the journey from ultraviolet to infrared. This extends the idea, alluded to above, that there should be a 'web' of integrable models linking the RG fixed points - in many cases, the trajectories making up this web are now seen to be just 
the boundaries of two-dimensional surfaces of integrability in the space of theories, swept out by the one-parameter families of staircase models.

Finally, we come to the sets of flows which will be the focus of our attention in the rest of this article. The homogeneous sine-Gordon (HSG) models will be introduced in greater detail below. It turns out that their RG behaviours combine various features of the flows discussed above in an interesting way. On the one hand, their deep UV limits are straightforwardly described as coset conformal field theories, with none of the subtleties seen in the cases of the staircase models. On the other hand, for suitable choices of the perturbing parameters, they can pass close by a number of other conformal field theories on their way to the infrared. Figure tuning of the parameters, it has been arranged that the fixed point $B$ has been missed, so only the conformal fixed points $A, C$ and $D$ are seen by the flow.
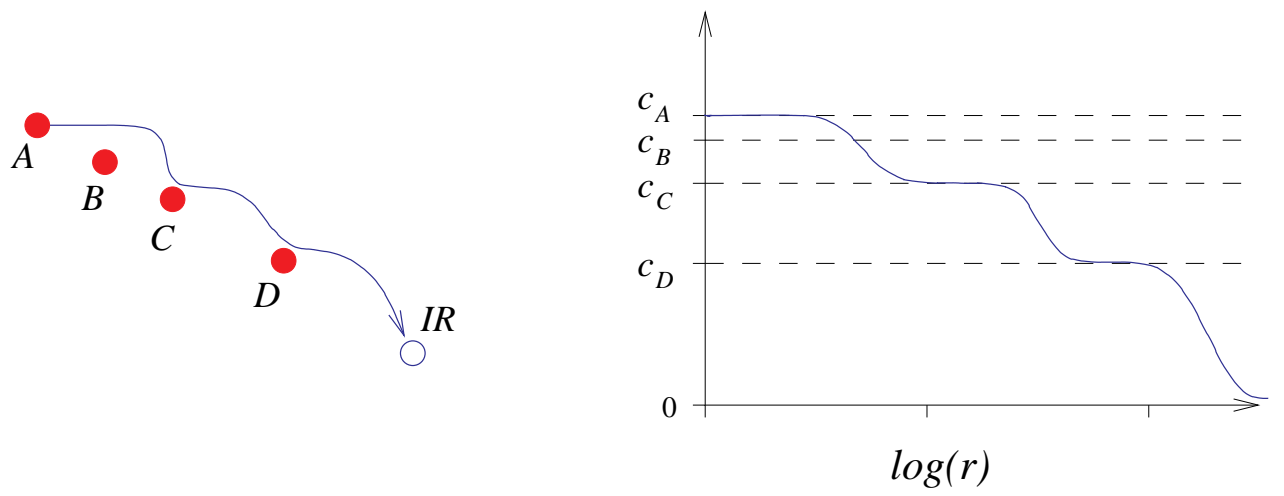

Figure 4: An HSG flow. The ultraviolet fixed point is $A$, so $c_{U V}=c_{A}$. Since the flow remains far from the fixed point $B$, there is no plateau in $c(r)$ at $c_{B}$. Labelling conventions as in figure

Since the HSG models have many parameters, there are many options for the flows, and considered together they sweep out whole manifolds of integrability in the space of theories. Our main aim in [i] w was to understand the possibilities in detail, and to relate them to the known properties of the models in their semiclassical limits. The TBA treatment of HSG models had previously been discussed in [1] $1 \overline{3}$, while an analysis of the flows

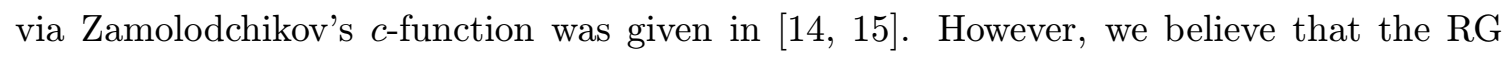
behaviour of general HSG models is considerably richer than had been suspected on the basis of the earlier work. In the rest of this article we shall outline some aspects of this story.

\section{The Homogeneous Sine-Gordon models}

After the rather discursive introduction, we now get down to specifics. The HSG models [1] are two-dimensional integrable quantum field theories that describe integrable perturbations of coset conformal field theories (CFTs) of the form $G_{k} / U(1)^{r_{g}}$, where $G$ is a simple compact Lie group with Lie algebra $g, k>1$ is an integer, and $r_{g}$ is the rank of $g$. Equivalently, they are integrable perturbations of the theory of level- $k G$-parafermions [i] $[\overline{1}]$, and can be viewed as the generalization of the perturbation of the $\mathbb{Z}_{k}$ parafermionic CFT by 


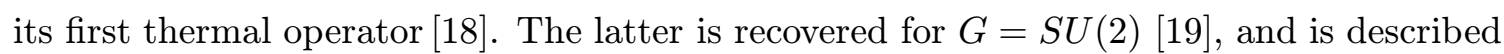
by the minimal $a_{k-1}$ factorised $S$-matrix [i2 $\left.{ }_{2}^{2} \overline{1}\right]$.

For $G \neq S U(2)$, the HSG models are examples of multiparameter deformations of CFTs. From this perspective, the models can be specified by actions of the form

$$
S_{\mathrm{HSG}}=S_{\mathrm{CFT}}-\mu \int d^{2} x \Phi,
$$

where $S_{\text {CFT }}$ denotes an action for the CFT of level- $k G$-parafermions, and $\Phi$ is a spinless primary field with conformal dimensions $\Delta_{\Phi}=\bar{\Delta}_{\Phi}=h_{g}^{\vee} /\left(k+h_{g}^{\vee}\right)$, with $h_{g}^{\vee}$ the dual Coxeter number of $g$. The field $\Phi$ lies in a multiplet in the unperturbed CFT, and a particular field $\Phi$ is determined by $2 r_{g}-2$ dimensionless parameters. Thus, in addition to the dimensionful parameter $\mu$, means that the theory depends on $2 r_{g}-1$ parameters, one of which can always be mapped onto the overall scale.

A more explicit definition of the models corresponding to perturbations of the coset $G_{k} / U(1)^{r_{g}}$ (which we shall refer to as the $G_{k}$-HSG in the following) is provided by the action [1]

$$
S_{\mathrm{HSG}}[\gamma]=S_{\mathrm{gWZW}}[\gamma]-\int d^{2} x V(\gamma)
$$

where $\gamma=\gamma(t, x)$ is a bosonic field taking values in the group $G, S_{\mathrm{gWZW}}$ is the gauged Wess-Zumino-Novikov-Witten (WZW) action corresponding to the coset $G / U(1)^{r_{g}}[\overline{2} \overline{2} \overline{1}]$, and the potential is

$$
V(\gamma)=\frac{m_{0}^{2}}{4 \pi \beta^{2}}\left\langle\Lambda_{+}, \gamma^{\dagger} \Lambda_{-} \gamma\right\rangle
$$

Here, $\langle$,$\rangle is the Killing form of g, m_{0}^{2}$ and $\beta^{2}$ are the bare mass scale and coupling constant, respectively, and $\Lambda_{ \pm}$are two arbitrary elements in the Cartan subalgebra of $g$ associated with the maximal torus $U(1)^{r_{g}}$, chosen to be not orthogonal to any root of $g$. Notice that the freedom to choose $\Lambda_{ \pm}$involves precisely $2 r_{g}-1$ adjustable parameters. In the quantum theory, the coupling constant becomes quantised, $\beta^{2} \simeq 1 / k+O\left(1 / k^{2}\right)$, and its role is played by the integer number $k>1$ known as the 'level'.

In this context, the $k \rightarrow \infty$ limit corresponds to both the weak-coupling (perturbative) and semiclassical regimes of $(\overline{2} . \overline{2})$, and this allows the $G_{k}-$ HSG models to be analysed at large $k$ using semiclassical techniques [i2 2in]. This has lead to conjectures for the mass spectra

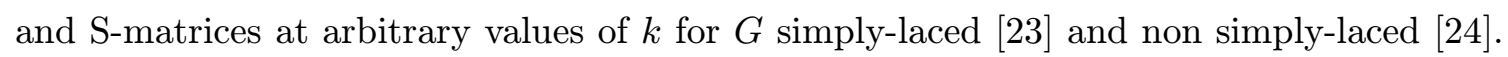
In the simply-laced cases, the S-matrices have now been checked using both TBA [1]

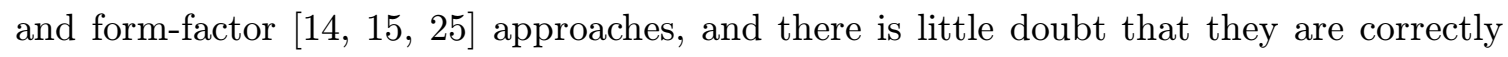
describing the perturbed parafermionic theories. However, there are aspects of these models that still have to be clarified.

One of them is the presence of unstable particles, which is a conjecture that also emerges from the semiclassical studies. Indeed, for the simply-laced $G_{k}-\mathrm{HSG}$ model, a total of $k-1$ particle-like states were identified for each positive root of $g$ in [i2 $\left[\begin{array}{l}2 \\ 2\end{array}\right]$, but only those corresponding to the roots of height 1 - the simple roots - turn out to be stable. 
In S-matrix theory, stable and unstable particles play completely different roles [i2 $\left.2 \bar{\sigma}_{i}^{\prime}\right]$, and it is only the particles associated with the simple roots whose existence is immediately confirmed by the checks on the exact S-matrices. Fortunately, there are physical observables, such as correlation functions and finite-size effects, where both types of particles are expected to play similar roles, setting the scales of crossover phenomena. This is because the effective behaviour of the system at a certain energy scale depends on the number of particle states which are effectively massless compared to that scale, irrespective of their stability. Examining the system at different scales thus provides a well-defined method to detect the existence of physical mass scales associated with both stable and unstable particles: at the mass scales corresponding to a physical particle state, a sharp change in the behaviour of the system should be observed, due to the decoupling of that particle.

The study of crossover phenomena for certain HSG models has already provided nonperturbative evidence of the existence of physical mass scales associated with the roots of heights 1 and 2. In particular, the TBA was applied to the study of finite-size effects in the $S U(3)_{k}-$ HSG models in [i] ${ }_{1}^{1} \overline{1}$. Moreover, the renormalisation group flow of Zamolodchikov's $c$-function was calculated in [1] 11 , 15$]$ for the $S U(N)_{2}$-HSG models, making use of the expansion of two-point correlation functions in terms of form factors. However, in [i] trace was found of any mass scale associated with the roots of height larger than 2 .

The main result of [i] $\left[\begin{array}{l}1 \\ 1\end{array}\right]$ is that the study of finite-size effects using the TBA technique indeed provides non-perturbative evidence for the presence of unstable particles states associated will all the roots of $g$, and at any value of $k$, large or small. Nevertheless, due to the nature of crossover phenomena, the study of finite-size effects does not allow to find the value of the corresponding mass scales with arbitrary precision; the most that one can ask is to pick up well-separated scales. Therefore, in order to understand properly the results of the TBA analysis, it is convenient to have a clue as to how many separated scales can be expected. In our case, this is provided by studying how many separated classical mass scales can be manufactured by varying the $2 r_{g}-1$ adjustable parameters; this will be addressed in the following section.

\section{Particle masses and classical mass scales}

The $S$-matrices proposed in [i2 $\overline{3}]$ for the simply-laced HSG models describe the scattering between solitonic particles labelled by two quantum numbers, $(i, a)$, where $i=1 \ldots r_{g}$ and $a=1 \ldots k-1$ for the $G_{k}$-HSG models. The mass of the particle $(i, a)$ is

$$
M_{a}^{i}=M m_{i} \mu_{a}
$$

where $M$ sets the overall mass scale, $m_{i}, i=1 \ldots r_{g}$, are $r_{g}$ arbitrary (non vanishing) relative mass scales attached to the nodes of the Dynkin diagram of $g$, and $\mu_{a}=\sin (\pi a / k)$ are the components of the Perron-Frobenius eigenvector of the $A_{k-1}$ Cartan matrix. In addition to the mass ratios of the stable particles, the S-matrix is sensitive to a further $r_{g}-1$ so-called resonance parameters $\sigma_{i j}=-\sigma_{j i}$, initially defined for each pair $\langle i, j\rangle$ of neighbouring nodes on the Dynkin diagram of $g$. These are most conveniently specified by 
assigning a variable $\sigma_{i}$ to each node of $g$ and setting $\sigma_{i j}=\sigma_{i}-\sigma_{j}$. This way, the $G_{k}$-HSG factorised $S$-matrix theory is determined by the $2 r_{g}-1$ parameters $\left\{m_{i}, \sigma_{i j}\right\}$.

Via the index $i$, each set of $k-1$ stable particles in the quantum theory is associated with a simple root $\vec{\alpha}_{i}$. In addition, classically, the theory exhibits sets of solitonic particlelike solutions associated with all of the other positive roots $\vec{\beta} \in \Phi^{+}$as well. Their mass scales can be written in a concise way as follows. Ignore for a moment the fact that $m_{i}$ and $\sigma_{i j}$ are parameters of the quantum theory, and define a couple of vectors in the weight space of $g$ by setting

$$
\vec{\lambda}_{ \pm}=\sum_{1}^{r_{g}} m_{i} e^{ \pm \sigma_{i}} \vec{\lambda}_{i}
$$

where the $\vec{\lambda}_{i}, i=1 \ldots r_{g}$, are the fundamental weights of $g$ and satisfy $\vec{\lambda}_{i} \cdot \vec{\alpha}_{j}=\delta_{i j}$. Then the relative mass scale of the particles associated with the positive root $\vec{\beta}$ is given by the formula

$$
m_{\vec{\beta}}^{2}=\left(\vec{\lambda}_{+} \cdot \vec{\beta}\right)\left(\vec{\lambda}_{-} \cdot \vec{\beta}\right)
$$

which reduces to $m_{i}^{2}$ for $\vec{\beta}=\vec{\alpha}_{i}$, as it should.

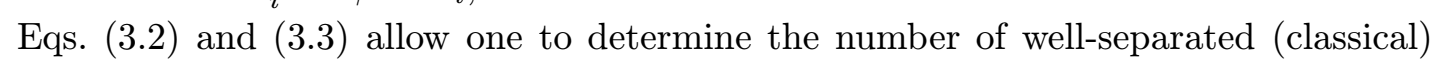
scales that can be constructed by tuning the adjustable parameters. Take a positive root $\vec{\beta}=\sum_{1}^{r_{g}} c_{i} \vec{\alpha}_{i}$, and substitute this expansion in (3.3. $\left.\overrightarrow{3}_{-1}^{-}\right)$. Then,

$$
m_{\vec{\beta}}^{2}=\sum_{i, j=1}^{r_{g}} c_{i} c_{j} m_{i} m_{j} e^{\sigma_{i}-\sigma_{j}}
$$

shows that all squared masses are expressed as linear combinations of the $r_{g}\left(r_{g}+1\right) / 2$ quantities

$$
2 m_{i} m_{j} \cosh \left(\sigma_{i}-\sigma_{j}\right), \quad i, j=1 \ldots r_{g}
$$

with coefficients $c_{i} c_{j}$ that are independent of the parameters $\left\{m_{i}, \sigma_{j}\right\}$, and are the squares of numbers of order one. Therefore, the model certainly has no more than $r_{g}\left(r_{g}+1\right) / 2$ separable mass scales, given by the numbers

$$
m_{i j}=\sqrt{m_{i} m_{j}} e^{\left|\sigma_{i}-\sigma_{j}\right| / 2}, \quad i, j=1 \ldots r_{g} .
$$

Notice that only in the $S U(N)_{k}$-HSG theories, for which $N=h_{g}=r_{g}+1$, is $r_{g}\left(r_{g}+1\right) / 2$ equal to the number of positive roots - in all other cases it is smaller. The number of separable mass scales that a classical HSG model can exhibit is therefore generally less than the number of positive roots. However, the scales ( the linear combinations $(\overline{3}-4)$, and it could be that some numbers from the set provided by (3.6i) never occur as the largest term in these sums for any choice of the values of the parameters, but rather are always swamped by other terms. Indeed, as explained in "iin, this is always the case for $g \neq a_{n}$. For all simply-laced Lie algebras, the maximal number of separable classical mass scales is given in table 


\begin{tabular}{|c||l|}
\hline \multicolumn{1}{|c||}{$g$} & Maximal number of separable scales \\
\hline \hline$a_{n}$ & $n(n+1) / 2$ \\
\hline$d_{n}$ & $n(n+1) / 2-1$ \\
\hline$e_{6}$ & $n(n+1) / 2-2, \quad n=6$ \\
\hline$e_{7}$ & $n(n+1) / 2-2, \quad n=7$ \\
\hline$e_{8}$ & $n(n+1) / 2-3, \quad n=8$ \\
\hline
\end{tabular}

Table 1: The maximal number of separable scales for the HSG models associated with the different simply-laced Lie groups.

\section{Detecting mass scales with the TBA equations}

We may now return to the quantum theory and analyse the scales generated in the TBA equations. It turns out that they show a very neat match with the separable classical scales. This leads to the conclusion that, in the quantum theory, there is also a mass scale associated with each positive root of $g$ and, remarkably, that it is given by Eqs. (3.2.) and (‥3.

Let us sketch the main features of the TBA analysis presented in [i]. The TBA equations for the HSG model have the standard form for a diagonal scattering theory, though care is needed in their derivation owing to the parity-breaking of the model [i] $1 \overline{3}$ in]. There is a pseudoenergy $\epsilon_{a}^{i}(\theta)$ for each of the $(k-1) \times r_{g}$ stable particles, while the $r_{g}$ mass scales $m_{i}$ influence the equations via $(k-1) \times r_{g}$ energy terms

$$
\nu_{a}^{i}(\theta)=M_{a}^{i} R \cosh \theta=m_{i} \mu_{a} r \cosh \theta
$$

where $\mu_{a}=\sin (\pi a / k)$ as before, and we have introduced a dimensionless overall crossover scale by

$$
r=M R
$$

Defining $L_{a}^{i}(\theta)=\log \left(1+\mathrm{e}^{-\epsilon_{a}^{i}(\theta)}\right)$, the system of TBA equations to be satisfied by the pseudoenergies is

$$
\epsilon_{a}^{i}(\theta)=\nu_{a}^{i}(\theta)-\sum_{b=1}^{k-1}\left(\phi_{a b} * L_{b}^{i}(\theta)+\sum_{j=1}^{r_{g}} I_{i j}^{g} \psi_{a b} * L_{b}^{j}\left(\theta-\sigma_{j i}\right)\right) .
$$

where the symbol ' $*$ ' denotes the usual rapidity convolution, $I^{g}$ is the incidence matrix of $g$, and the definition of the TBA kernels can be found in [1] $\left.{ }^{1} \overline{1}\right]$. They are of the same form as the kernels defining other TBA systems which had previously arisen in the contexts of

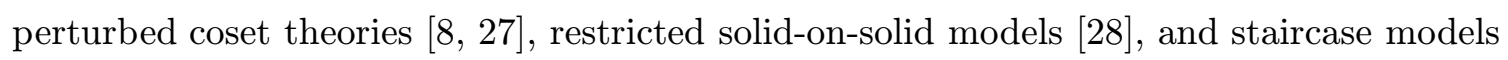

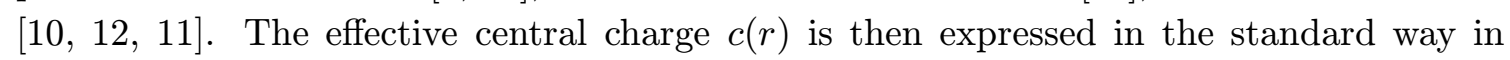
terms of the energy terms and the solutions to the TBA equations:

$$
c(r)=\frac{3}{\pi^{2}} \sum_{i=1}^{r_{g}} \sum_{a=1}^{k-1} \int_{-\infty}^{+\infty} d \theta \nu_{a}^{i}(\theta) L_{a}^{i}(\theta) .
$$


Note that, in this multiparameter case, $c(r)$ depends not only on $r$, but also on the relative mass scales and the resonance parameters: $c(r)=c\left(r ; m_{i}, \ldots, m_{r_{g}}, \sigma_{1}, \ldots, \sigma_{r_{g}}\right)$.

The limiting value of $c(r)$ in the $r \rightarrow 0$ limit corresponds, at least in unitary cases such as these, to the central charge of the conformal field theory describing the deep UV limit of the theory. For the HSG theories this quantity was calculated in [i] $\overline{3}_{-1}$, with the result

$$
\lim _{r \rightarrow 0} c(r)=\frac{k-1}{k+h_{g}} h_{g} r_{g}
$$

where $h_{g}$ is the Coxeter number of $g$. This holds for any fixed choice of the relative mass scales $0<m_{i}<+\infty$ and the resonance parameters $-\infty<\sigma_{i}<+\infty$. In other words, $c(r)$ tends to the central charge of the $G_{k} / U(1)^{r_{g}}$ coset CFT in the deep UV limit. In the opposite, $r \rightarrow \infty$, limit, $c(r)$ tends to zero, as expected for a massive theory.

However, this is not the only information hidden inside the TBA equations. For intermediate values of $r$, depending on the values taken by the parameters $\left\{m_{i}\right\}$ and $\left\{\sigma_{i}\right\}$, the scaling function $c(r)$ can show the characteristic staircase pattern mentioned in the introduction, indicating a renormalisation group flow which passes close to a number of other fixed points. In contrast to Zamolodchikov's original staircase model [9.9]

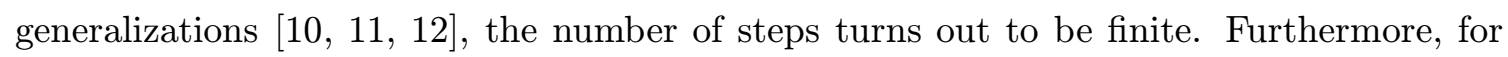
the HSG models all aspects of the staircase pattern can be understood physically, as a consequence of the decoupling of those particles that are effectively heavy at the relative energy scale fixed by the temperature $R^{-1}$, be they stable or unstable. This was already observed for the $S U(3)_{k}$-HSG models in [i] these cases were too simple for our purposes. The analysis presented in [i] applies to generic (simply-laced) $G_{k}$-HSG models, and follows a line of argument used originally to

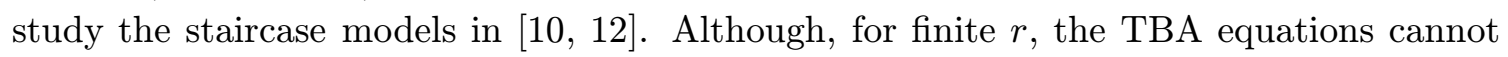
be solved exactly, this method allows a full understanding of the staircase pattern to be gained, subject only to some mild assumptions about the form of the solutions to (4.3). In fact, these assumptions are no more severe than those usually made in the analysis of the UV limit of standard TBA systems. In any case, the resulting predictions have been checked numerically in a number of particular cases.

The picture derived in [i] $\left[\begin{array}{l}1 \\ 1\end{array}\right]$ is based on the following properties of the TBA equations. First of all, due to the dominance of the energy terms $\nu_{a}^{i}(\theta)$,

$$
L_{a}^{i}(\theta) \approx 0 \quad \text { for } \quad|\theta| \gg-\log \left(\frac{2}{m_{i} r}\right), \quad a=1 \ldots k-1 .
$$

Second, the kernels $\phi_{a b}(\theta)$ and $\psi_{a b}(\theta)$ are in a well-defined sense local [i] $\left.10 \overline{0}\right] ;$ i.e., for real values of $\theta$, they are peaked about $\theta=0$, and fall off exponentially to become effectively zero outside a region of order one. This implies that the pseudoenergy $\epsilon_{a}^{i}\left(\theta_{0}\right)$ is influenced by the value of the energy term $\nu_{a}^{i}(\theta)$ at $\theta=\theta_{0}$, and by the pseudoenergies $\epsilon_{b}^{j}(\theta)$ at $\theta \approx \theta_{0}-\sigma_{j i}$, for all $j$ such that $I_{i j}^{g} \neq 0$.

An immediate consequence of eq. (14. $\left.\bar{L}_{i}^{i}\right)$ is that the pseudoenergies $\epsilon_{a}^{i}(\theta)$ do not contribute to the value of $c(r)$ for $2 r^{-1} \ll m_{i}$. Actually, in this regime, the TBA system corresponding to the $G_{k}$-HSG models truncates to the system associated to the $G_{k}^{[i]}$-HSG 
model, where we denote by $G^{[i]}$ the (semisimple) Lie group associated with the Dynkin diagram obtained by removing the node $i$ from the Dynkin diagram of $g$. Therefore, and provided that the mass scale $m_{i}$ is well-separated from any other scale in the system, the effective behaviour changes at $2 r^{-1} \approx m_{i}$. Physically, it corresponds to the decoupling of all the particles (stable and unstable) associated with the roots $\vec{\beta}$ such that $\vec{\beta} \cdot \overrightarrow{\lambda_{i}} \neq 0$.

The remaining expected changes in the value of the finite finite-size scaling function are found by studying whether the shifted convolution terms in the TBA equations (4.3) can be neglected of not for particular values of $r$, again as a consequence of eq. ('4.6i). The result can be summarised as follows [i]. Take two arbitrary nodes $k \neq l$ on the Dynkin diagram of $g$, and consider the unique chain of nodes joining them; i.e.,

$$
\left\{i_{p}, p=1 \ldots n\right\}, \quad \text { with } \quad i_{1}=k, i_{n}=l,
$$

and $\left\{i_{p}, i_{p+1}\right\}$ being neighbouring nodes for $p=1 \ldots n-1$. Then, the values of the pseudoenergies $\epsilon_{a}^{k}(\theta)$ and $\epsilon_{b}^{l}(\theta)$ are independent, for any $a, b=1 \ldots k-1$, when the value of $r$ is in the range

$$
m_{k l} \gg 2 r^{-1} \gg m_{i_{p} i_{q}}, \quad \forall p, q \in\{1 \ldots n\} \quad \text { with } \quad(p, q) \neq(1, n),
$$

where the numbers $m_{i j}$ are defined by eq. $\left(\overline{3} \cdot \overline{6}_{i}\right)$, but now as a function of the (quantum) $S$-matrix parameters. Conversely, those pseudoenergies do depend on each other for

$$
2 r^{-1} \gg m_{i_{p} i_{q}}, \quad \forall p, q \in\{1 \ldots n\} .
$$

This way, and provided that the scale $m_{k l}$ is well-separated from the other scales associated with this chain of nodes, the value of $c(r)$ will change abruptly at $2 r^{-1} \simeq m_{k l}$. These changes correspond to the decoupling of all the particles (stable and unstable) associated with the roots $\vec{\beta}$ such that both $\vec{\beta} \cdot \vec{\lambda}_{k} \neq 0$ and $\vec{\beta} \cdot \vec{\lambda}_{l} \neq 0$. When ' $k$ ' and ' $l$ ' are themselves neighbouring nodes on the Dynkin diagram of $g$, the corresponding changes of behaviour

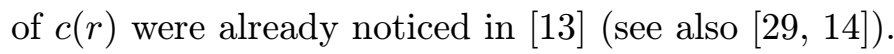

Therefore, the study of the TBA equations leads to the conclusion that the form of the scaling function $c(r)$ will be characterised by the value of the mass scales defined by eqs. $\left(\overline{3} \cdot \overline{2}_{i}^{\prime}\right)$ and $\left(\overline{3} \cdot \overline{3}_{i}^{\prime}\right)$, which we repeat here:

$$
\begin{aligned}
& m_{\vec{\beta}}^{2}=\left(\vec{\lambda}_{+} \cdot \vec{\beta}\right)\left(\vec{\lambda}_{-} \cdot \vec{\beta}\right), \\
& \vec{\lambda}_{ \pm}=\sum_{1}^{r_{g}} m_{i} e^{ \pm \sigma_{i}} \vec{\lambda}_{i}, \quad \vec{\lambda}_{i} \cdot \vec{\alpha}_{j}=\delta_{i j}
\end{aligned}
$$

where $\vec{\beta}$ is any positive root of $g$, and $\left\{m_{i}, \sigma_{i}\right\}$ are the $S$-matrix parameters. The scaling function $c(r)$ becomes nearly constant in the regions where $2 r^{-1}$ is between two consecutive mass scales, but far away from their actual values. Equivalently, $c(r)$ develops a plateau when the values of two of the scales provided by (40i) are well-separated and there is no other scale between them. This way, $c(r)$ is predicted to exhibit a staircase pattern with a finite number of plateaux, whose maximum number depends on the Lie group $G$ and is

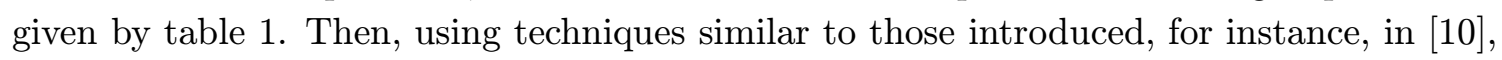




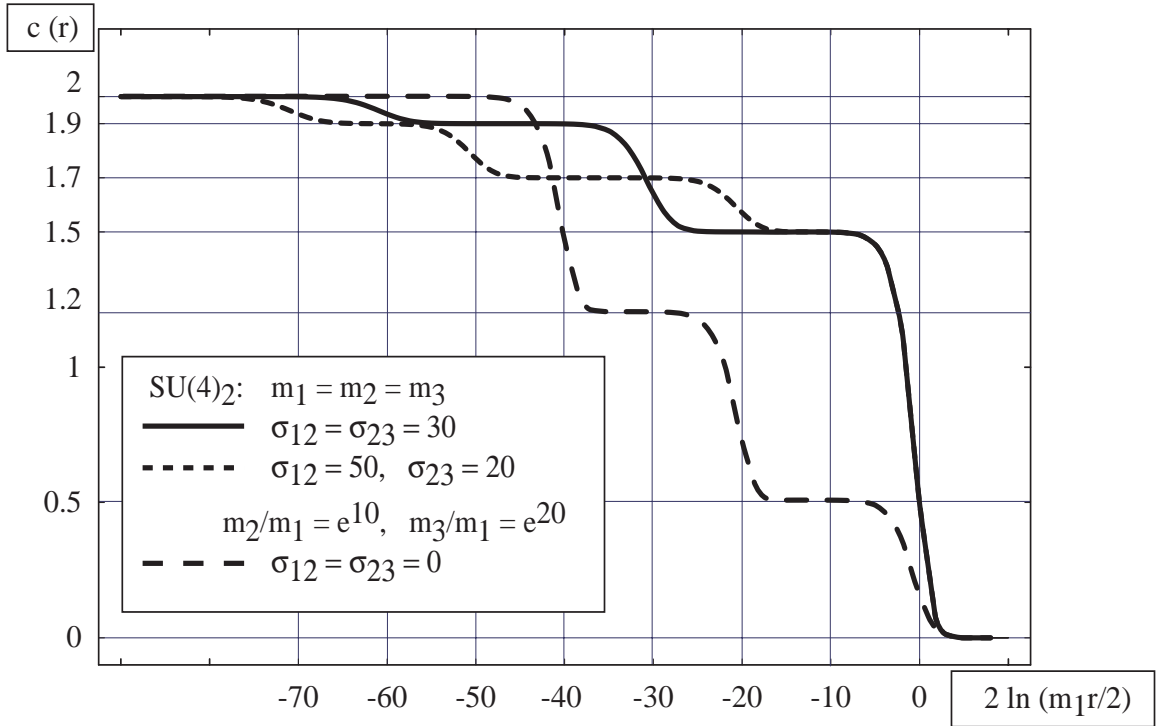

Figure 5: The TBA scaling function for the $S U(4)_{2} / U(1)^{3}$ HSG model.

it is possible to calculate the value of $c(r)$ on these plateaux, and thereby map out the RG flows. Further details will be given in [i].

Finally, we remark that for the HSG models there is an alternative way to calculate the plateau values of $c(r)$, using the physical interpretation of the staircase pattern as a consequence of the decoupling of those particles that are effectively heavy at the relative energy scale fixed by the temperature $r^{-1}$, together with the Lagrangian description provided by $(2.2])$. In addition, this procedure leads to a well-defined conjecture for the relationship between Lagrangian and $S$-matrix parameters. Once more, we refer the interested reader

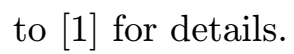

The results summarised in the previous paragraphs rely on assumptions about the behaviour of the solutions to the TBA equations, which, though well-motivated, have not been rigorously established. Therefore, we have subjected them to some independent checks. In particular, the TBA equations ( of cases. Figures ${ }_{-1}^{15}$, and $\overline{6}_{1}^{\prime}$ show some numerical results for $G=S U(4), S U(5)$, and $S O(8)$, with $k=2$ and different choices for the resonance parameters and the mass scales. In order to illustrate the resulting patterns of flows, we have collected some of them in the appendix. Remarkably, they can be understood as composite flows made of elementary massive and massless flows between conformal field theories. Results for $G=S U(3)$ and $k=2,3,4$ were presented in [1] $\left.{ }_{1}^{1}\right]$. In all the cases considered, the agreement with our predictions is excellent. In particular, they confirm that the form of the scaling function $c(r)$ depends on the value of the scales given by (4.10i), associated with all the positive roots of $g$. 


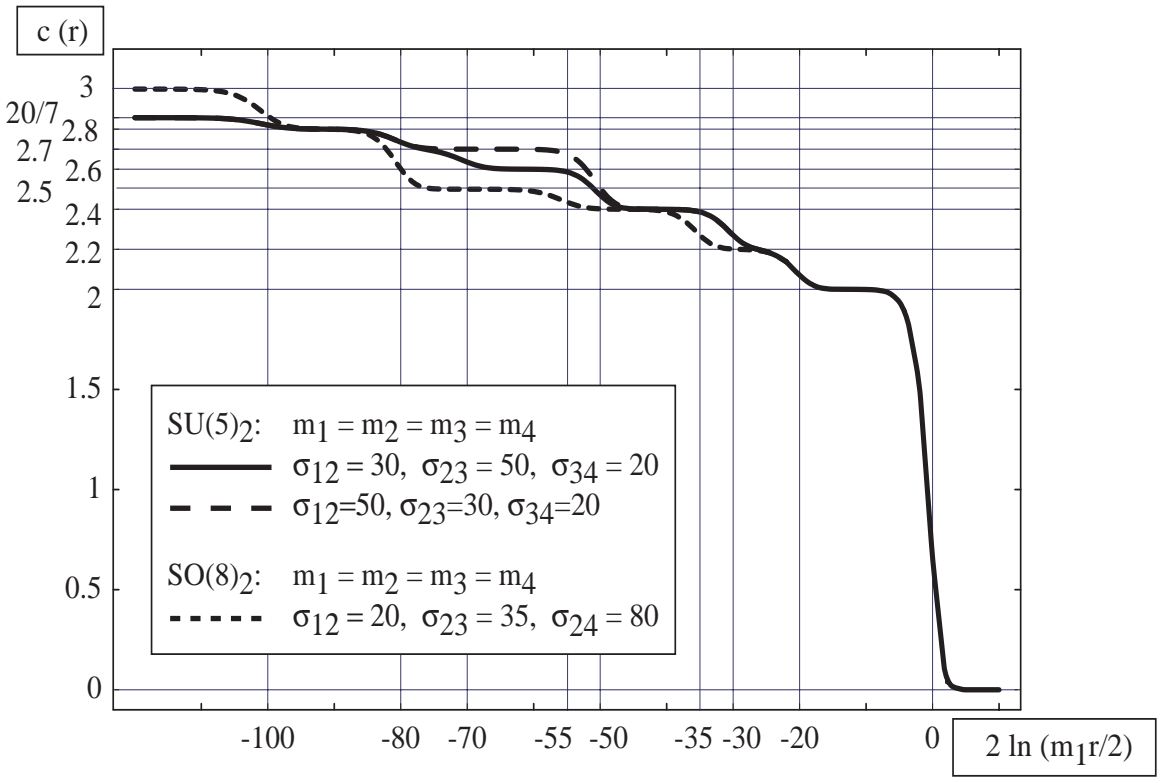

Figure 6: The TBA scaling function for the $S U(5)_{2} / U(1)^{4}$ and $S O(8)_{2} / U(1)^{4} \mathrm{HSG}$ models.

\section{Summary and discussion}

To sum up, the main result of [i] in the study of finite-size effects using the TBA provides non-perturbative evidence for the existence of an independent mass scale associated with each positive root of $g$ in the $G_{k}$-HSG models, although this way only well-separated scales can be directly seen. This is in agreement with the results of [1] $\left.{ }^{1}\right]$ and [1] for the $S U(3)_{k}-$ HSG models, but disagrees with the results of [1] ${ }_{1}^{1} \bar{y}_{1}$ for $S U(N)_{2}$ with $N \geq 4$. This work is based on the form-factor approach, and failed to detect any of the $(N-3)(N-2) / 2$ mass scales associated with the roots of $a_{N-1}$ of height larger than 2. The resolution of this discrepancy could reveal some novel features of the form-factor method.

Let us finish by mentioning three open questions about the HSG models raised by our work and not addressed in this article. First of all, and despite the fact that these models are described by diagonal $S$-matrix theories, the influence of the extra unstable particles gives the HSG models a much richer structure of RG flows than had previously been claimed, giving them a rather universal status which unifies the simplest flows between CFTs within a common structure. This should help towards a better understanding of the nature of the $G_{k}$-HSG models, particularly at small values of $k$, where descriptions in terms of coupled minimal models have recently been investigated [ijo $\left.\overline{0}_{1}^{\prime}\right]$.

The second concerns the recent observation of novel classical breather solutions in the complex Sine-Gordon (CSG) model [3] $\overline{1} \overline{1}$. It turns out that these solutions can be understood in terms of the known spectrum of the model [i] ${ }_{1}^{1} \overline{1}$, but their presence was nonetheless unexpected. The CSG model describes the $S U(2)_{k}$-HSG model at large $k$, and the semiclassical spectrum of the generic $G_{k}$-HSG models was constructed by embedding 


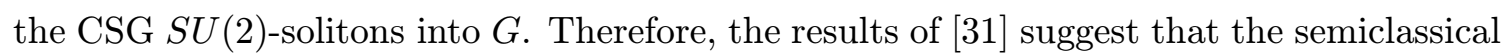
analysis could still hide some more surprises, although our results confirm to a large extent the semiclassical picture for the HSG models derived in [2]2i]

Finally, a feature of the HSG models which has played very little direct role in our analysis so far is the fact that they generally break parity. It would be very interesting to pick up signs of this in the finite-size behaviour of the theories. Probably the best place to start looking would be in the spectrum of excited states. The TBA technique can also be applied to excited states $\left[\overline{3} \overline{2}_{1}^{1}, \overline{3} \overline{3} \overline{3}\right.$, and we hope to have more to say on this issue in the future.

\section{Acknowledgments}

One of us (JLM) would like to thank the organisers of the workshop for their kind hospitality, and congratulate the IFT people on their $50^{\text {th }}$ anniversary. PED thanks the Universidad de Santiago de Compostela for hospitality and financial support while this work was in progress. We are grateful to Pascal Baseilhac, Peter Bowcock, Aldo Delfino and Roberto Tateo for valuable discussions. The work of JLM is supported partially by Xunta de Galicia (PGDIT00-PXI-20609), and MCyT and FEDER (BFM2002-03881 and FPA2002-01161). We also thank the EC Commission for financial support via the FP5 Grant HPRN-CT-2002-00325.

\section{A. Staircase patterns as RG flows.}

In this appendix, we describe explicitly the flow of effective field theories corresponding to some of the cases whose numerical solutions were presented in figures $\underline{15}_{-1}^{1}$ and $\overline{6}$.

\section{A.1 $S U(4)_{k}-$ HSG models.}

We consider first a case with vanishing resonance parameters, and mass scales chosen such that $m_{1} \ll m_{2} \ll m_{3} .{ }^{1}$ Then, the scaling function exhibits 3 plateaux corresponding to the regions $2 r^{-1} \gg m_{3}$ (the deep UV limit), $m_{2} \ll 2 r^{-1} \ll m_{3}$, and $m_{1} \ll 2 r^{-1} \ll m_{2}$, before it reaches the massive region for $2 r^{-1} \ll m_{1}$ where $c(r)$ vanishes. Within each region, $c(r)$ equals the central charge of the following parafermionic coset CFT's:

$$
(U V) \frac{S U(4)_{k}}{U(1)^{3}} \stackrel{m_{3}}{\longrightarrow} \frac{S U(3)_{k}}{U(1)^{2}} \stackrel{m_{2}}{\longrightarrow} \frac{S U(2)_{k}}{U(1)} \stackrel{m_{1}}{\longrightarrow} \text { Massive }(I R) .
$$

Next, let us consider a second case with non-vanishing resonance parameters chosen such that

$$
\sigma_{12} \gg \sigma_{23} \gg 0 \text { and } m_{1}=m_{2}=m_{3},
$$

\footnotetext{
${ }^{1}$ In the following, we will use the (standard) conventions of [34] for numbering the nodes of the Dynkin diagram of $g$.
} 
which corresponds to

$$
\begin{aligned}
& m_{\vec{\alpha}_{1}+\vec{\alpha}_{2}+\vec{\alpha}_{3}} \simeq m_{13} \\
& m_{\vec{\alpha}_{1}+\vec{\alpha}_{2}} \simeq m_{12}, \quad m_{\vec{\alpha}_{2}+\vec{\alpha}_{3}} \simeq m_{23}, \\
& m_{\vec{\alpha}_{1}}=m_{\vec{\alpha}_{3}}=m_{\vec{\alpha}_{3}}=m_{1}
\end{aligned}
$$

where

$$
m_{13} \gg m_{12} \gg m_{23} \gg m_{1}
$$

The resulting flow is

$$
\begin{aligned}
\left(\frac{S U(4)_{k}}{U(1)^{3}}\right)^{<2>} & \stackrel{m_{13}}{\longrightarrow}\left(\frac{S U(3)_{k}}{S U(2)_{k} \otimes U(1)} \otimes \frac{S U(3)_{k}}{U(1)^{2}}\right)^{<\frac{19}{10}>} \\
& \stackrel{m_{12}}{\longrightarrow}\left(\frac{S U(3)_{k}}{U(1)^{2}} \otimes \frac{S U(2)_{k}}{U(1)}\right)^{<\frac{17}{10}>} \\
& \stackrel{m_{23}}{\longrightarrow}\left(\left[\frac{S U(2)_{k}}{U(1)}\right]^{\otimes 3}\right)^{<\frac{3}{2}>} \stackrel{m_{1}}{\longrightarrow} \text { Massive }<0>
\end{aligned}
$$

where the superscript $<>$ provides the central charge of the corresponding coset CFT for level $k=2$, to simplify the comparison with the numerical results presented in figures $\overline{\bar{p}_{1}}$ and $\overline{\underline{6}} \bar{v}_{r}^{\prime}$

\section{A.2 $S U(5)_{k}-$ HSG models.}

First, consider the following choice of parameters

$$
\sigma_{23} \gg \sigma_{12} \gg \sigma_{34} \gg 0 \text { and } m_{1}=m_{2}=m_{3}=m_{4}
$$

which leads to

$$
\begin{aligned}
& m_{\vec{\alpha}_{1}+\vec{\alpha}_{2}+\vec{\alpha}_{3}+\vec{\alpha}_{4}} \simeq m_{14} \\
& m_{\vec{\alpha}_{1}+\vec{\alpha}_{2}+\vec{\alpha}_{3}} \simeq m_{13}, \quad m_{\vec{\alpha}_{2}+\vec{\alpha}_{3}+\vec{\alpha}_{4}} \simeq m_{24}, \\
& m_{\vec{\alpha}_{1}+\vec{\alpha}_{2}} \simeq m_{12}, \quad m_{\vec{\alpha}_{2}+\vec{\alpha}_{3}} \simeq m_{23}, \quad m_{\vec{\alpha}_{3}+\vec{\alpha}_{4}} \simeq m_{34} \\
& m_{\vec{\alpha}_{1}}=m_{\vec{\alpha}_{3}}=m_{\vec{\alpha}_{3}}=m_{\vec{\alpha}_{4}}=m_{1}
\end{aligned}
$$

where

$$
m_{14} \gg m_{13} \gg m_{24} \gg m_{23} \gg m_{12} \gg m_{34} \gg m_{1} .
$$

The resulting flow is

$$
\left(\frac{S U(5)_{k}}{U(1)^{4}}\right)^{<\frac{20}{7}>} \stackrel{m_{14}}{\longrightarrow}\left(\frac{S U(4)_{k}}{S U(3)_{k} \otimes U(1)} \otimes \frac{S U(4)_{k}}{U(1)^{3}}\right)^{<\frac{14}{5}>}
$$




$$
\begin{aligned}
& \stackrel{m_{13}}{\longrightarrow}\left(\frac{S U(4)_{k}}{S U(2)_{k} \otimes U(1)^{2}} \otimes \frac{S U(3)_{k}}{U(1)^{2}}\right)^{<\frac{27}{10}>} \\
& \stackrel{m_{24}}{\longrightarrow}\left(\left[\frac{S U(3)_{k}}{S U(2)_{k} \otimes U(1)}\right]^{\otimes 2} \otimes \frac{S U(3)_{k}}{U(1)^{2}}\right)^{<\frac{13}{5}>} \\
& \left.\stackrel{m_{23}}{\longrightarrow}\left(\left[\frac{S U(3)_{k}}{U(1)^{2}}\right]^{\otimes 2}\right)^{<\frac{12}{5}>}\right)_{m_{12}}^{\longrightarrow}\left(\frac{S U(3)_{k}}{U(1)^{2}} \otimes\left[\frac{S U(2)_{k}}{U(1)}\right]^{\otimes 2}\right)^{<\frac{11}{5}>} \\
& \stackrel{m_{34}}{\longrightarrow}\left(\left[\frac{S U(2)_{k}}{U(1)}\right]^{\otimes 4}\right)^{<2>} \stackrel{m_{1}}{\longrightarrow} \text { Massive }<0>.
\end{aligned}
$$

Notice that the flow exhibits 7 plateaux, while, according to table for the $S U(5)_{k}$-HSG models is 10 . The difference is due to the fact that we have chosen the 4 stable mass scales to be equal, which means that 3 of them are not separated.

Second, consider another choice of parameters such that

$$
\begin{aligned}
& \sigma_{12} \gg \sigma_{23} \gg \sigma_{34} \gg 0, \quad \sigma_{23}+\sigma_{34}=\sigma_{12}, \\
& \text { and } m_{1}=m_{2}=m_{3}=m_{4},
\end{aligned}
$$

which corresponds to

$$
m_{14} \gg m_{13} \gg m_{24}=m_{12} \gg m_{23} \gg m_{34} \gg m_{1} .
$$

The resulting flow is

$$
\begin{aligned}
\left(\frac{S U(5)_{k}}{U(1)^{4}}\right)^{<\frac{20}{7}>} & \stackrel{m_{14}}{\longrightarrow}\left(\frac{S U(4)_{k}}{S U(3)_{k} \otimes U(1)} \otimes \frac{S U(4)_{k}}{U(1)^{3}}\right)^{<\frac{14}{5}>} \\
& \stackrel{m_{13}}{\longrightarrow}\left(\frac{S U(4)_{k}}{S U(2)_{k} \otimes U(1)^{2}} \otimes \frac{S U(3)_{k}}{U(1)^{2}}\right)^{<\frac{27}{10}>} \\
& \stackrel{m_{24}=m_{12}}{\longrightarrow}\left(\left[\frac{S U(3)_{k}}{U(1)^{2}}\right]^{\otimes 2}\right)^{<\frac{12}{5}>} \\
& \stackrel{m_{23}}{\longrightarrow}\left(\frac{S U(3)_{k}}{\left.U(1)^{2} \otimes\left[\frac{S U(2)_{k}}{U(1)}\right]^{\otimes 2}\right)^{<\frac{11}{5}>}}\right. \\
& \stackrel{m_{34}}{\longrightarrow}\left(\left[\frac{S U(2)_{k}}{U(1)}\right]^{\otimes 4}\right)^{<2>} \stackrel{m_{1}}{\longrightarrow} \text { Massive }<0>.
\end{aligned}
$$

This case exhibits one plateau less that the previous one, which is a consequence of the fact that the scales $m_{24}$ and $m_{12}$ are not separated for this choice of parameters. 


\section{A.3 $S O(8)_{k}-$ HSG models.}

We shall only consider the choice of parameters

$$
\sigma_{12}=20, \quad \sigma_{23}=35, \quad \sigma_{24}=80, \quad \text { and } \quad m_{1}=m_{2}=m_{3}=m_{4},
$$

which corresponds to

$$
\begin{aligned}
& m_{\vec{\alpha}_{1}+2 \vec{\alpha}_{2}+\vec{\alpha}_{3}+\vec{\alpha}_{4}}, m_{\vec{\alpha}_{1}+\vec{\alpha}_{2}+\vec{\alpha}_{3}+\vec{\alpha}_{4}}, m_{\vec{\alpha}_{1}+\vec{\alpha}_{2}+\vec{\alpha}_{4}} \simeq m_{14} \\
& m_{\vec{\alpha}_{2}+\vec{\alpha}_{4}}, m_{\vec{\alpha}_{2}+\vec{\alpha}_{3}+\vec{\alpha}_{4}} \simeq m_{24} \\
& m_{\vec{\alpha}_{1}+\vec{\alpha}_{2}+\vec{\alpha}_{3}} \simeq m_{13} \\
& m_{\vec{\alpha}_{2}+\vec{\alpha}_{3}} \simeq m_{23}, \quad m_{\vec{\alpha}_{1}+\vec{\alpha}_{2}} \simeq m_{12} \\
& m_{\vec{\alpha}_{1}}=m_{\vec{\alpha}_{3}}=m_{\vec{\alpha}_{3}}=m_{1}
\end{aligned}
$$

where

$$
m_{14} \gg m_{24} \gg m_{13} \gg m_{23} \gg m_{12} \gg m_{1}
$$

The resulting flow is

$$
\begin{aligned}
& \left(\frac{S O(8)_{k}}{U(1)^{4}}\right)^{<3>} \stackrel{m_{14}}{\longrightarrow}\left(\frac{S U(4)_{k}}{S U(3)_{k} \otimes U(1)} \otimes \frac{S U(4)_{k}}{U(1)^{3}}\right)^{<\frac{14}{5}>} \\
& \stackrel{m_{24}}{\longrightarrow} \equiv\left(\frac{S U(4)_{k}}{U(1)^{3}} \otimes \frac{S U(2)_{k}}{U(1)}\right)^{<\frac{5}{2}>} \\
& \stackrel{m_{13}}{\longrightarrow}\left(\frac{S U(3)_{k}}{S U(2)_{k} \otimes U(1)} \otimes \frac{S U(3)_{k}}{U(1)^{2}} \otimes \frac{S U(2)_{k}}{U(1)}\right)^{<\frac{12}{5}>} \\
& \stackrel{m_{23}}{\longrightarrow}\left(\frac{S U(3)_{k}}{U(1)^{2}} \otimes\left[\frac{S U(2)_{k}}{U(1)}\right]^{\otimes 2}\right)^{<\frac{11}{5}>} \\
& \stackrel{m_{12}}{\longrightarrow}\left(\left[\frac{S U(2)_{k}}{U(1)}\right]^{\otimes 4}\right)^{<2>} \stackrel{m_{1}}{\longrightarrow} \text { Massive }^{<0>} .
\end{aligned}
$$

In this case the flow exhibits 6 plateaux, which agrees with the maximal number quoted in table $i_{-1}^{-1}$ which is 9 , taking into account that we have chosen the 4 stable mass scales to be equal.

\section{References}

[1] P. Dorey and J.L. Miramontes, Mass scales and crossover phenomena in the Homogeneous Sine-Gordon Models, preprint DCPT-02/81, in preparation.

[2] A.B. Zamolodchikov, Sov. Physs. JETP Lett. 433 (1986) 730-732,

[3] A.B. Zamolodchikov, 'isov. Jucl. Phys. 46 $(1987)$ 1090-1096i. 
[4] A.B. Zamolodchikov, Sov Phys. JETP Lett. 46 (1987) 160-164 Int. J. Mod. Phys. A 4! -

[5] J.L. Cardy, Physs. Rev. Lett. 60 (1988) 2709-27111

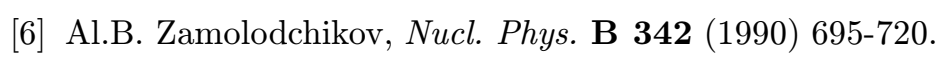

[7] H.W.J. Blöte, J.L. Cardy and M.P. Nightingale, 'Phys. Rev. Lett. $\mathbf{5} \overline{\mathbf{6}}(\overline{1} \overline{9} \overline{8} \overline{6}) \overline{7} \overline{4} 2-\overline{4} \overline{4}$; I. Affleck, 'Phys. Rev. Lett. $\mathbf{5} 6$ - (1986) 746-748'

[8] Al.B. Zamolodchikov, №vcl. $\bar{P}$ hys. B $358-1991) 524-546$.

[9] Al.B. Zamolodchikov, Resonance Factorized Scattering and Roaming Trajectories, preprint ENS-LPS-335 (1991).

[10] P. Dorey and F. Ravanini, Int. J. Mod.Phys. A 8 (1993) $873-893$.

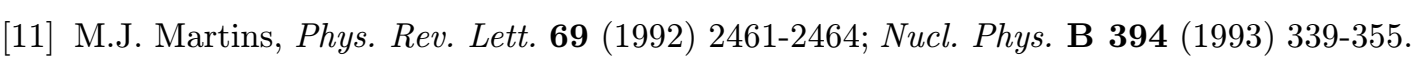

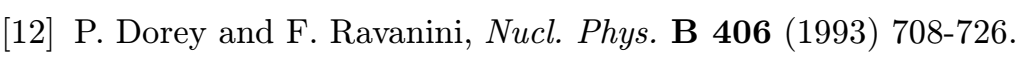

[13] O.A. Castro-Alvaredo, A. Fring, C. Korff and J.L. Miramontes, - - -

[14] O.A. Castro-Alvaredo and A. Fring, PPhys. Rev. D 63 3 $(2001) 021701(\bar{R})^{\prime}$.

[15] O.A. Castro-Alvaredo and A. Fring, iPhys. Rev. D 64 (2001) 085007.

[16] C.R. Fernández-Pousa, M.V. Gallas, T.J. Hollowood and J.L. Miramontes, 'Nucl. Phys. Bi'

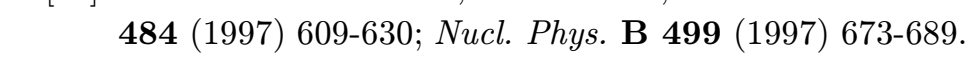

[17] D. Gepner, 'Nucl. Phys. B 290_(1987) 10-24' Phys. Lett. B 222 $19892207-212$.

[18] V.A. Fateev, 'Int. J. Mod. Phys. A 6 (1991) $210 \overline{9}-2 \overline{1} 32$

H. de Vega and V.A. Fateev, Int. J. Mod. Phys. A 6 (1991) 3221-323,

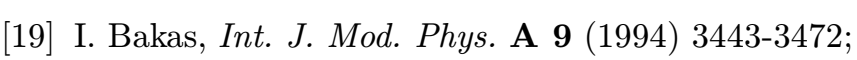

Q-H. Park, Phys. Lett. B $\mathbf{3 2 8}(1994) 329-336$

A.M. Tsvelik, INucl. Phys. B 305 (1988) $675-684$.

[20] R. Köberle and J.A. Swieca, 'Phys. Lett.

[21] E. Witten, 'Comm. Math. Phys. $14 \overline{4}(1992) 189-212$ '

K. Gawedzki and A. Kupiainen, 'Nucl. Phys. B 320 (1989) 625-668.

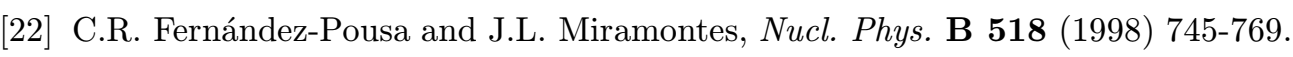

[23] J.L. Miramontes and C.R. Fernández-Pousa, Phys. Lett. $\bar{B}^{-} \overline{4} \overline{2}(2000) 392-40 \overline{1}_{1}^{\prime}$

[24] C. Korff, Phys. Lett. B 501 (2001) 289-296.

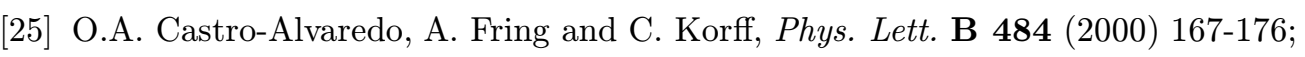

O.A. Castro-Alvaredo and A. Fring, Nucl. Phys. B 604 (2001) 367-390.

[26] R.J. Eden, P.V. Landshoff, D.I. Olive and J.C. Polkinghorne, The analytic S-matrix, Cambridge Univ. Press (1966);

R.G. Newton, Scattering theory of waves and particles, McGraw-Hill (1966).

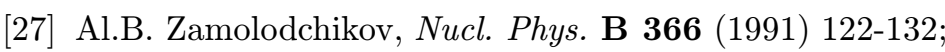

F. Ravanini, 'Phys. Lett. B 282

F. Ravanini, R. Tateo and A. Valleriani, Int. J. Mod. Phys. Ä 8 (1993) 1707-17̄2

R. Tateo, Int. J Mod. Phys. A 10 (1995) 1357-1376. 
[28] A. Kuniba and T. Nakanishi, ${ }^{M} \bar{M} \bar{d}$. Phys. Lett. A $\overline{7}(\overline{1} \overline{9} \overline{2}) \overline{3} 4 \overline{8} \overline{3} 4 \overline{4}$

A. Kuniba, T. Nakanishi and J. Suzuki, 'Mod. Phys. Lett. A 8 (1993) 1649-1660̈.

[29] J.L. Miramontes, in proceedings of Non-perturbative Quantum Effects 2000, Paris (2000), eds. D. Bernard et al., PRHEP-tmr2000/036, 'hep-th/0010012.

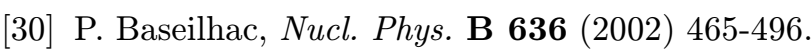

[31] P. Bowcock and G. Tzamtzis, The complex Sine-Gordon model on a half line, Durham U. preprint DCPT-02-21, hep-th/0203139.

[32] V.V. Bazhanov, S.L. Lukyanov and A.B. Zamolodchikov, №cl. Phys. B 489 (1999) $487-531 \overline{4}$ hep-th/9607099.

[33] P. Dorey and R. Tateo, iNucl. Phys. B 482 (1996) 639-659, hep-th/9607167.

[34] V.G. Kac, Infinite dimensional Lie algebras (3 ${ }^{\text {rd }}$ ed.), Cambridge University Press (1990). 